\title{
LA EVOLUCION DEL DIPTONGO o EN BEOCIO
}

\begin{abstract}
While the usual inscriptional spelling for the outcomı of the diphthong ot is $\mathrm{Y}$ in most towns of Boeotia, it frequently appears as EI in some late inscriptions in Chaeronea, Lebadea and Coronea. According to current opinion, these facts suggest an iotacistic development $/ \mathrm{oi} />\mid \overline{\ddot{g}} />/ \ddot{\mathrm{u}} />/ \overline{\mathrm{i}} /$, supposedly in anticipation of the evolution of the diphthong o $o$ in Late koเví and Early Byzantine Greek. In our paper we will argue that this interpretation is based on insufficient grounds, and we will present an alternative hypothesis, viz., that Boeotian EI spellings for Common Greek o $\iota$ do not correspond to an [i] pronunciation, but rather - as would be expected to an [ẹ]] sound. Consequently, we maintain that the Boeotian diphthong o had developed into an $/ \overline{0} /$ vowel ( $\mathrm{Y}$ in the inscriptions) by the end of the fourth century B.C., which subsequently became $/ \overline{\mathrm{e}} /$ (EI) in the aforementioned towns. Thus the evolution of Boeotian o is not comparable with that of Late Greek ol.
\end{abstract}

\section{A Don Ricardo Castresana}

0. Una de las características más destacables del beocio la constituye el desarrollo de su sistema vocálico, en el que, como es sabido, se anticipan algunas de las innovaciones que, durante la época de la koiné helenística y bizantina, transformaron el sistema de vocales del ático clásico en el del griego moderno'.

1. Con respecto a la evolución diacrónica del diptongo /oi /, los datos proporcionados por las inscripciones beocias suelen interpretarse del modo siguiente: la tendencia de /oi/ a la asimilación (paralela a la de los diptongos /ei//, /ai /, /oụ/) empieza a manifestarse desde el s. $v$ a.C. cuando en algunas inscripciones arcaicas, especialmente en Tanagra, pero también en otras localidades boecias, una notación OE (que debe co-

' La prioridad cronológica del beocio seria mucho menor si se aceptase la polémica datación de Teodorsson (1974), que sitúa el comienzo de la mayor parte de los cambios en ático clásico. 
rresponder a una realidad fonética [oẹ]) sustituye ocasionalmente a OI. En las inscripciones en alfabeto reformado del siglo Iv, donde OI es con diferencia la notación más frecuente, la aparición de una nueva grafía $\mathrm{Y}$ en lugar de $\mathrm{OI}$ permite deducir que el diptongo /oi/ había experimentado - al menos para algunos hablantes del dialecto beocio - una contracción ${ }^{2}$. Dado que en el alfabeto jónico el signo $\mathrm{Y}$ servía para notar

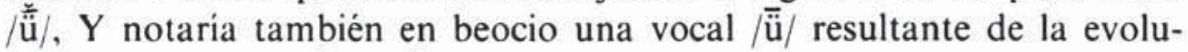
ción $/ \mathrm{oi} />[\mathrm{oe}]>[\overline{\ddot{\alpha}}]>/ \overline{\mathrm{u}} /$. El uso de $\mathrm{Y}$ se generaliza hasta convertirse progresivamente en norma a partir de $c a .250$, pero sin que la grafia arcaizante OI llegue a desaparecer por completo.

A fines del siglo III y durante el s. II, en las inscripciones del área más occidental de Beocia (Queronea, Lebadea y Coronea), fundamentalmente actas de manumisión ${ }^{3}$, se comprueba que la grafia $\mathrm{Y}$ comienza a alternar con EI incluso dentro de un mismo documento: p.ej., Roesch y Fossey (1978a) n. ${ }^{\circ} 4$ (Queronea, fin. s. III) $14 \varepsilon \pi$ हांuma, pero 6-7 y 11

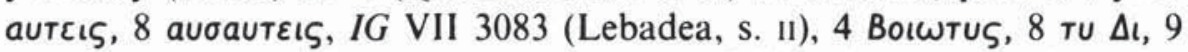

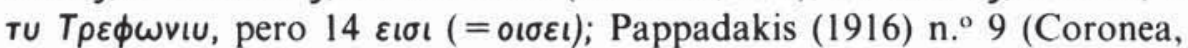

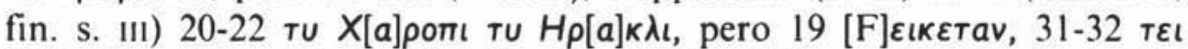
$\beta \varepsilon \iota \lambda o \mu \varepsilon v \varepsilon \iota$ (dat. sg.). La distribución de $\mathrm{Y}$ y EI para los resultados de /oi/ en las localidades citadas es la siguiente: en Coronea $\mathrm{Y}$ aparece en 13 ocasiones $(76,4 \%)$ frente a 4 veces EI $(23,5 \%)$; en Lebadea, 16 veces Y $(59,2 \%)$ y 11 EI $(40,7 \%)$; sólo en Queronea los casos de Y (23 veces, $45 \%$ ) se ven superados por los de EI ( 28 veces, $54,9 \%$ ). Aunque los datos son demasiado escasos para poder obtener conclusiones estadisticamente fiables, parece que la proporción de EI frente a $\mathrm{Y}$ crece progresivamente desde la localidad situada más al este, Coronea, a la más occidental, Queronea, donde puede localizarse el epicentro del cambio. La grafía histórica OI sólo se emplea para un nombre propio en una

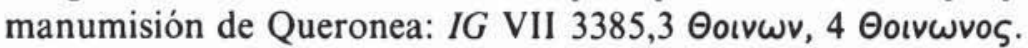

Esos mismos textos atestiguan esporádicamente formas con I en lugar de EI (cf., p.ej., en la última inscripción citada, $24 \lambda \varepsilon \iota \tau \omega \rho \gamma \iota \varepsilon v$ por $\lambda \varepsilon เ T \omega \rho \gamma \varepsilon(\mu \varepsilon v)$, que indican que la /ẹ/ (continuadora de la ${ }^{*} \bar{e}$ del gr. común y de la $\bar{e}$ secundaria resultante de alargamientos y contracciones) estaba confundiéndose con /i//. Basándose en este hecho, los estudiosos

\footnotetext{
2 Ante vocal, con una sola excepción (IG VII 2864, decreto honorifico de Coro-

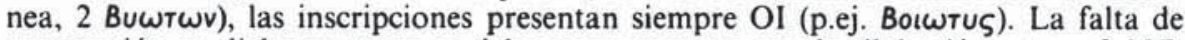
contracción en dicho contexto se debe seguramente a que la silabación no era [oi.. V], sino [o.jV].

${ }^{3}$ A las recogidas por Dittenberger en el vol. VII de las Inscriptiones Graecae (Berlín 1982), hay que añadir catorce manumisiones procedentes de Coronea publicadas por Pappadakis (1916: pp. 217-24 y 269), otra de la misma localidad (Roesch y Fossey 1978b), asi como nueve de Queronea (Roesch y Fossey 1978a).
} 
explican las grafias EI por $\mathrm{Y}$ de las localidades señaladas como grafias inversas en las que EI serviria para notar la /i/ resultante de la deslabialización de $/ \tilde{\mathrm{u}} /(</ \mathrm{oi} /)$. La evolución de $/ \mathrm{oi} /$ en beocio anticiparia, pues, los cambios de la koiné tardia /oi / > / / $/$ / en el s. II d.C. (Threatte 1980: p. 337) y, tras la desaparición de la cantidad vocálica como rasgo fonológico, / $/$ / $>/$ i/ en el s. x (cf. Browning 1983: pp. 56-57).

Entre los estudios y manuales de Dialectologia griega que suscriben esta interpretación cabe citar a Meister (1882: pp. 237-38) ${ }^{4}$, Meyer (1896: p. 185), Thumb (1909: p. 224), Buttenwieser (1911: p. 29), Hirt (1912: p. 161), Buck (1955: $\S 30)^{5}$, Thumb-Scherer (1959: $\left.\S 236,8 \mathrm{c}\right)$, Blümel (1982: pp. 65-66), quien afina la cronología de la documentación y señala la presencia de grafías EI en manumisiones de Coronea que habian pasado desapercibidas a autores anteriores, y Brixhe et alii (1985: pp. 301-302) ${ }^{6}$. Son también partidarios de una equivalencia $\mathrm{Y}=$ $/ \overline{\mathrm{u}} /$, aunque no toman en consideración las grafias EI de las localidades citadas, Beermann (1876: pp. 41-42), Sturtevant (1940: p. 52), Bartoněk (1966: pp. 31, 119-20), Allen (1972: pp. 63-64, 77) ${ }^{7}$. Lejeune (1972: $\S 243$ ), Rix (1976: p.47). Schmitt (1977: p. 70).

Blaß (1881: pp. 610-11) y Sadée (1903: pp. 68-71) coinciden con los anteriores en atribuir a $\mathrm{Y}$ una pronunciación [ū]. Sin embargo, ambos autores ponen en entredicho la hipótesis de que EI en lugar de $Y$ sea un recurso para notar $/ \mathbf{i} /$ (cf. infra, $\S 3.1$ ). Blaß cree que EI sería el reflejo

\footnotetext{
4 Meister propone vı (sc. [üi]) como etapa intermedia en el cambio /oi $/>/ \mathrm{u} /$. Pero, según ya señalaba Beermann (1876: pp. 41-42), oponiéndose a una propuesta similar de Curtius y Dietrich, la etapa [üi] queda descartada por las grafias arcaicas

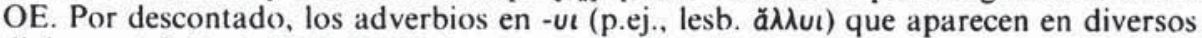
dialectos (cf. Buck 1955: $\$ 132,4$ ) no proceden fonéticamente de formas más antiguas en -o como creia Meister.

5 Buck sostiene explícitamente que $\mathrm{Y}$ en beocio debe tomarse como una notación

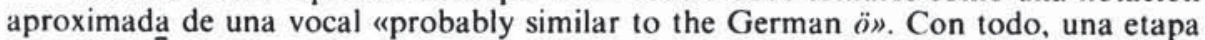
posterior $/ \ddot{\mathrm{u}} /$ parece deducirse de manera implicita al menos para Lebadea y Coronea de sus palabras: "the spelling $\varepsilon \iota$ [en lugar de $u$ ]... indicat[es] the further progress of the sound to $i$ ).

${ }^{6}$ Brixhe et alii (1985) sólo se refieren al desarrollo del diptongo /oi/ en el dat. sg. temático. Como observa con acierto Blümel (1982: p. 65), la generalización de Y en este dativo no se produce hasta comienzos del s. II (es decir, casi medio siglo más tarde que en el resto de los datos), lo que induce a creer que la contracción del diptongo /oi / primario antecede a la de /oi// secundario del dativo, que procede de un diptongo con primer elemento largo /ọj/. Esta diferencia cronológica en la generalización de Y, sumada a otros argumentos (vid. Méndez Dosuna 1985: pp. 413-63) permite explicar, pese al escepticismo de Brixhe et alii (1985), el dat. sg. temático en -o del beocio y otros dialectos griegos como resultado de la abreviación fonética del diptongo - $\omega \iota$ sin necesidad de recurrir a la explicación alternativa de carácter morfológico, que lo hace continuador del antiguo locativo (tipo át. olkoı).

7 Allen aduce como argumento corroborativo del cambio $/ \bar{o} />/ \bar{u} /$ el posible paralelismo con la confusión de $/ \overline{\mathbf{e}} /(</$ ei $/)$ con $/ \overline{\mathbf{i}} /$.
} 
de un sonido intermedio entre [ữ] y [ẹ]], es decir: [ọ̄]. Para Sadée (1903: p. 71), el digrafo EI representaria «sono $i$ admixtum aliquid [...], quod accurate definire non possumus».

También se aparta de la communis opinio Ruipérez (1956: p. 80) al considerar que la vacilación entre $\mathrm{Y}$ y $\mathrm{EI}$ es de naturaleza puramente gráfica. Ambas grafias serían intentos imperfectos de transcribir un fonema / $\overline{\mathrm{u}} /$ que quedaba a medio camino entre $/ \overline{\mathrm{u}} /$ (notada en ocasiones $\mathrm{Y}$ siguiendo la tradición local) e /i// (notada EI).

Bechtel (1921: p. 224) interpreta Y como notación de una vocal [ọ̄], pero no especifica qué criterio le permite alcanzar tal conclusión y rechazar el posible valor [ü]. Sobre la grafia EI prefiere no definirse: "Welcher Laut mit EI bezeichnet worden ist, steht dahin.»

Por último, Schwyzer (1939: pp. 194-95), aunque sin apoyar su afirmación con ningún argumento y sin especificar el valor fonético atribuible a Y, sostiene, frente a todos los autores citados, que EI es transcripción de /ệ/. Extrañamente, Schwyzer cita - ¿en apoyo de esta hipótesis? - a Blaß (1881), para quien EI nota [ö], a Meister (1882) y Thumb (1909), partidarios de la equivalencia EI = [1], y a Bechtel (1921), que, como hemos visto, no se pronunciaba al respecto.

2. En lo que sigue, intentaremos demostrar que las equivalencias $Y$ $=/ \overline{\mathrm{u}} / \mathrm{EI}=/ \mathrm{i} /$ postuladas por los partidarios de la communis opinio obligan a forzar la interpretación natural de los datos de las inscripciones: estos se explican de modo más coherente si se admite con Bechtel que $\mathrm{Y}$ representa /ö/ y que EI es - de acuerdo con Schwyzer - transcripción de $/ \bar{e} /$.

2.1. En primer lugar, no resulta convincente la solución postulada por Blaß (1881), pues, teniendo en cuenta que $\mathrm{Y}$ notando el resultado de $/ \mathrm{oi} / \mathrm{se}$ atestigua mucho antes que El, habría que admitir un proceso [ü] > [ọ̄], lo que parece poco plausible.

2.2. No parece tampoco probable que $\mathrm{Y}$ y EI sean meras variantes notacionales de la vocal / $\overline{\mathrm{u}} /$ como sugiere Ruipérez (1956). Si esto fuera así, esperaríamos a fortiori que la vacilación entre ambas notaciones no se produjese en una zona tan limitada ya que grafias iotacisticas con I en lugar de EI se documentan en todo el territorio beocio. En consecuencia, el empleo de EI en lugar de $\mathrm{Y}$ debe considerarse sintoma de un proceso fonético en Queronea, Lebadea y Coronea.

2.3. El principal argumento esgrimido por los partidarios de las 
equivalencias $\mathrm{Y}=/ \overline{\mathrm{u}} / \mathrm{y} \mathrm{EI}=/ \overline{\mathrm{i}} /$ son las grafias $\mathrm{I}$ por $\mathrm{EI}$ a que hemos hecho referencia ${ }^{8}$. Es preciso destacar, sin embargo, que el número de errores de este tipo en las manumisiones de las tres localidades citadas es muy reducido: Pappadakis (1916: $n^{\circ}{ }^{2}$ ), (Coronea, fin. s. III) 13-14 $\lambda \varepsilon เ T \omega \rho[\gamma] \iota \tau \omega$ (la notación correcta EI aparece en 14 ocasiones) ${ }^{9}$; IG VII

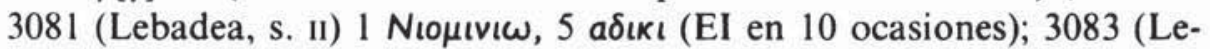
badea, s. II) $24 \lambda \varepsilon เ T \omega \rho \gamma \iota \mu \varepsilon v$ (EI en 13 ocasiones). Tres ejemplos más en manumisiones de Queronea parecen indicar que el cambio $/ \bar{e} />/ \mathbf{i} /$ se veía favorecido por el contacto con [j] ([jẹ̄] > [jī] quizá > [i]): Roesch

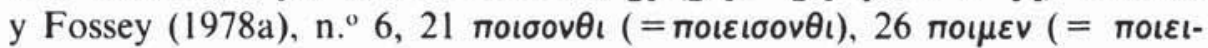
$\mu \varepsilon v)$, n. ${ }^{\circ} 9,8$ a conservada (o restaurada por analogía con el resto del paradigma) en una manumisión de Coronea, Roesch y Fossey (1978b) 8 Hрак $\lambda \varepsilon \varepsilon \iota$ ha evolucionado a $/ \overline{1} /$, resultado que atestiguan otras actas de la misma localidad, Pappadakis (1916) n. ${ }^{\circ} 2,8, \mathrm{n} .^{\circ} 3,61, \mathrm{n} .^{\circ} 6,32$ Нрак $\lambda_{l}$, frente a [ẹ] en los números 4, 74 y 10, 48 Нрак $\lambda \varepsilon \iota$.

Algunos casos se registran en manumisiones de Queronea (s. II) re-

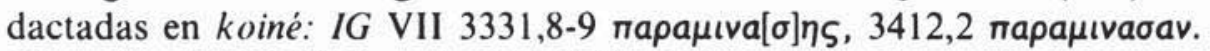

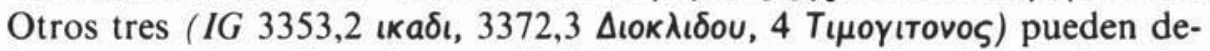
berse a una interferencia con el dialecto puesto que las formas beocias equivalentes tienen $/ \mathbf{i} /(</$ ei $/)$.

3. Todos estos errores ponen de manifiesto que en beocio /ệ (escrita EI) estaba cerrándose y confundiéndose con /i/, aunque lo más probable es que el proceso no hubiese llegado todavía a su término. Pero esto no es razón suficiente para atribuir a EI en lugar de $\mathrm{Y}$ un valor $/ \mathbf{i} /$.

3.1. En primer lugar, infunde serias sospechas el hecho de que I, el

* Sólo tomaremos en cuenta los casos de I por EI ante consonante o en posición final de palabra. Un único ejemplo ante vocal, que se registra en una manumisión

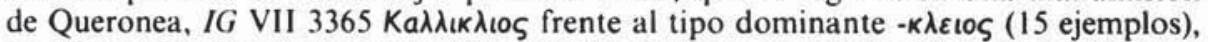
no afecta a nuestra argumentación. En dicho contexto, /ei/ tendia a convertirse en [j] al igual que las vocales $/ \mathrm{e} / \mathrm{,} / \mathrm{i} /$.

9 Es probable que la forma $x ı \lambda ı a s$ en otra manumisión de Coronea, Pappadakis (1916: n. 7,72 ), se deba explicar como un préstamo de la koiné en lugar del epicóri-

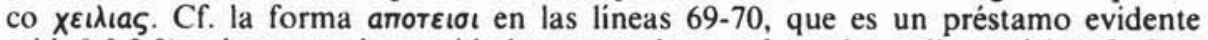

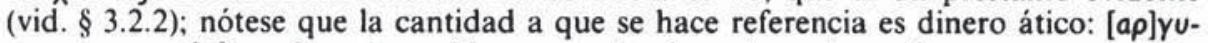

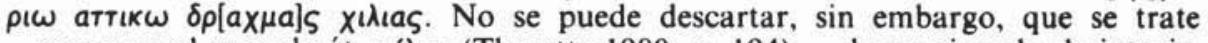
- como en el caso de át. xi 1 iıı (Threatte 1980: p. 194) - de un ejemplo de iotacismo temprano (cf. también infra, n. 11).

${ }^{10}$ A juicio de Brixhe (1985: p. 377) la pérdida de yod en aфıтı se debe a una especie de disimilación (?) inducida por la /î/ adyacente. 
signo empleado normalmente para /i/, no alterne nunca con $\mathrm{Y}$ y EI cuando se trata de notar la supuesta /i/ continuadora de /oi/ a través de una etapa / $\overline{\mathrm{u}} /$, en Queronea, Lebadea y Coronea. No resulta verosímil que los beocios de las localidades citadas hayan ideado una notación especial con el único fin de distinguir gráficamente la /i/ secundaria procedente de $/ \overline{\mathrm{u}} /$ de la $/ \bar{i} /$ ya existente en el dialecto. Esta objeción, por si misma suficiente para poner en tela de juicio la equivalencia $\mathrm{EI}=/ \mathbf{i} /$, fue planteada ya - con poco eco entre los estudiosos posteriores - por Blaß (1881: p. 611) y por Sadée (1903: p. 71, "cur tandem Boeoti, si modo saeculo altero $i$ pro $u ̈$ pronuntiabant, in scriptura EI adeo persisterent, cur ne semel quidem EI cum I conmutarent?»). Sin embargo, como hemos expuesto más arriba, las soluciones que ofrecen estos dos autores tampoco son satisfactorias.

3.2. En segundo lugar, cuando EI se interpreta como una grafia inversa de /i/, se está dando por supuesto de forma más o menos tácita que tales grafias inversas se documentan en las inscripciones para notar /i/ de otro origen. Vamos a ver inmediatamente que esto no es así y que los pretendidos ejemplos de grafia inversa EI notando /i/ en las manumisiones de Queronea, Lebadea y Coronea son ilusorios.

3.2.1. La forma $\delta a v \varepsilon ı \omega$ en un contrato de Lebadea ( $I G$ VII 3054,4) no es probatoria. La palabra es un tecnicismo tomado de la koiné y, como tal, presenta sistemáticamente EI en todas las inscripciones beocias en que aparece (vid. Buttenwieser 1911: p. 73).

3.2.2. Otro tecnicismo de la koiné es el verbo anoteı kis (1916: n. $\left.{ }^{\circ} 7,70\right)$ (Coronea, fin. s. III). Su origen extraño al dialecto se evidencia en el resultado $T$ de la labiovelar ${ }^{*} k^{y}$ ante $e$; la forma auténticamente dialectal aparece en el acuerdo financiero entre Tisbe y

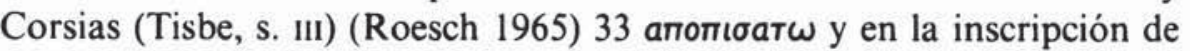
Nicareta (Orcómeno, fin. s. III), IG VII 3172, VI 8 потапопьбarw (cf. la combinación de los dos términos en cuestión ibid. en un pasaje redacta-

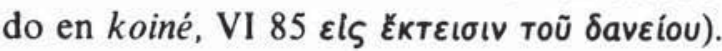

3.2.3. Al influjo de los modelos ortográficos de la koiné hay que atribuir también el uso de EI para dos nombres propios en dos actas de

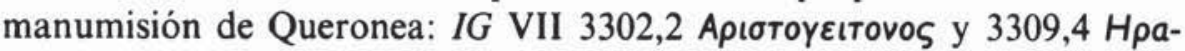
$\kappa \lambda \varepsilon เ \delta a v$ (cf. ibid. $1 \mu \eta v o \varsigma$ ). No es éste el único indicio en los textos epigráficos de esta época que delata la progresiva pérdida de terreno del dialecto ante el avance de la koiné: $\zeta$ en lugar de $\delta$, пара у ката en lu- 
gar de las variantes apocopadas пар у кат, etc. (vid. Buttenwieser 1911, passim; Buck 1955: p. 238)"

3.2.4. Al lado de las formas esperables con I atestiguadas en Leba-

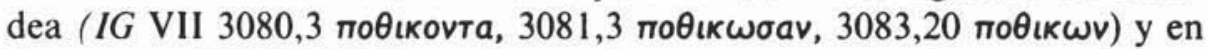

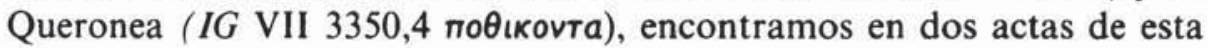

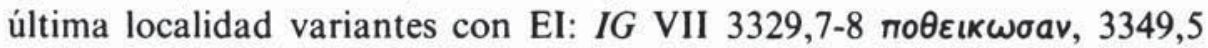

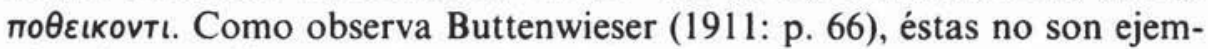
plos de notación EI de la /i / del dialectal ï $\kappa \omega$, sino que resultan de una adaptación a la fonética beocia de la forma de la koiné equivalente $\ddot{\eta} \kappa \omega$. Cf. $\kappa т \varepsilon เ \sigma \omega v \theta \eta$ en una acta de Coronea (Pappadakis 1916: n. ${ }^{\circ} 13$,

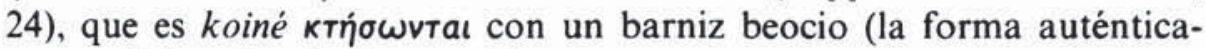
mente dialectal seria * $\pi a \sigma \omega v \theta \eta)$.

3.2.5. También el influjo de la koiné es perceptible en el uso de la desinencia $-\varepsilon \iota$ de dativo con el nombre del dios Sérapis en cuatro actas de Queronea de las redactadas en dialecto ( $I G$ VII 3305,3; 3313,5 Eapa-

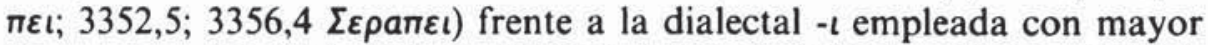
frecuencia: $I G$ VII 3301,$3 ; 3303,3 ; 3304,3 ; 3307,2 ; 3315,5 ; 3319,4$; 3329,$7 ; 3346,4 ; 3348,4 ; 3349,3-4 ; 3350,3-4 ; 3351,3 ; 3360,7 ; 3375,2$;

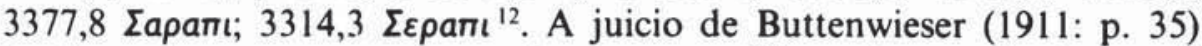
las formas en $-\varepsilon \iota$ son meras variantes ortográficas ( $s c$. grafias inversas) de - . Esta suposición la fundamenta en la frecuencia con que EI de la koiné suplanta a la I dialectal desde finales del siglo III en las inscripciones. Pero, dado que, como intentamos hacer ver, no existen ejemplos irrefutables de grafias inversas EI por I en las manumisiones en dialecto, parece más razonable aceptar que la desinencia de dativo sg. $-\varepsilon \iota$, que se documenta en época tardía también en otros muchos dialectos que no conocen el cambio /ẹ/ $/>/ \bar{i} /$ (Buck 1955: § 109,2), responde al para-

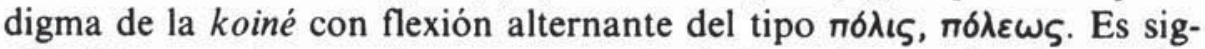
nificativo a este respecto que $-\varepsilon \iota$ sea la desinencia que domina en las

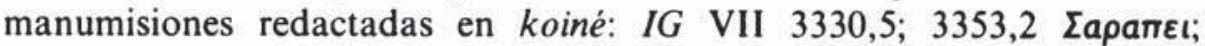

\footnotetext{
"Buttenwieser (1911: p. 40) considera que la forma de la condicional $\varepsilon$, atestiguada con mucha frecuencia en las inscripciones, es un rasgo de la koiné que suplanta a la dialectal $\eta(<a \imath)$. Es posible, no obstante, que $\varepsilon \iota$ proceda fonéticamente de $\eta$ por un cierre temprano de $/ \check{\S} / ;$ cf. el iotacismo temprano de la conjunción condicional en chipriota, donde $\bar{e}$ alterna con $\bar{l}$ (vid. Thumb-Scherer $1959 ; \S 274,5$ ).

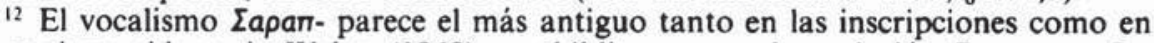
los papiros; vid., p.ej., Weber (1968) con bibliogr.; para la variación $\Sigma a p a n-\sim \Sigma \varepsilon$ pan- en ático, Threatte (1980: pp. 122-24).
} 


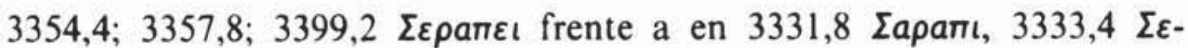
parı ${ }^{13}$.

3.2.6. En otras actas de Queronea la divinidad a la que quedan consagrados los manumisos es Ártemis Ilitia. Este epiteto (originariamente el nombre de una divinidad independiente asimilada más tarde a Ártemis) muestra un gran número de variantes. Es en el vocalismo de la segunda silaba donde se observa la variación I $\sim$ EI que aquí nos interesa. Con I encontramos: Roesch y Fossey (1978a), n. ${ }^{\circ} 5,5, \mathrm{n} .{ }^{\circ} 6,16$,

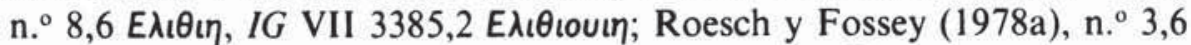

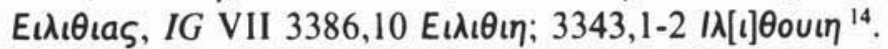

Presentan EI: Roesch y Fossey (1978a), n. ${ }^{\circ} 7,5$ E $\lambda \varepsilon \varepsilon \theta i \eta ; n .{ }^{\circ} 1,6$, n. ${ }^{\circ}$

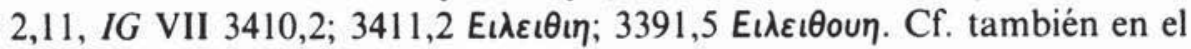

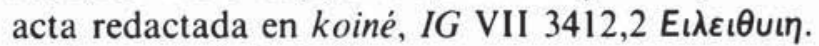

La variedad de formas con que aparece el teónimo en Queronea ${ }^{15}$ sólo puede causar una sorpresa relativa. Éstas y otras variantes se atestiguan en todo el ámbito griego. Aunque su etimología es todavía dis-

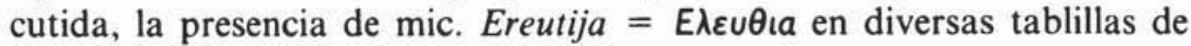

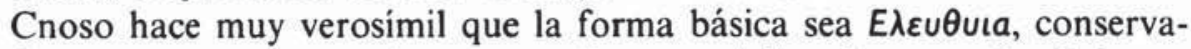
da en cretense. Las variantes con diptongo /ei/ en la segunda sílaba re-

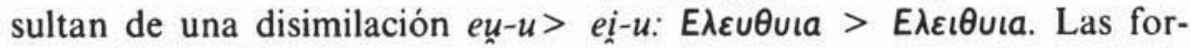
mas del tipo $E \iota \lambda \varepsilon \imath \theta$ - se explican como homerismos con alargamiento métrico o, mejor, por asimilación del vocalismo de la primera sílaba al de la segunda ${ }^{16}$.

Si la disimilación $e u-u>e i-u$ se produjo en beocio antes del s. v a.C., lo que no se puede verificar por falta de datos en inscripciones arcaicas, el diptongo /ei/ de la segunda sílaba se habrá confundido con el diptongo /ei/ primario y habrá evolucionado a /i/. En este caso, las formas con I serían las antiguas y las variantes con EI deberian ponerse en relación con la koiné. Pero, teniendo en cuenta que la variación I $\sim$ EI se documenta para el teónimo en cuestión en otras áreas dialectales, es perfectamente posible que en Beocia coexistiesen desde siempre formas

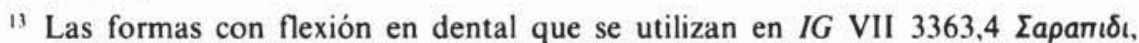

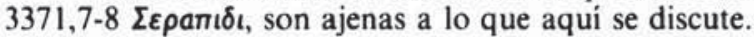

14 Inscripción muy fragmentaria en la que no puede descartarse la reconstrucción $[E] \iota \lambda[\iota]$ Oouı .

is Análoga variedad de formas encontramos en las inscripciones votivas de Tespias publicadas por A. Plassart, $B C H$ 50, 1926, p. 383 ss., nos. 25-37. Todas ellas, a excepción de los nos. $33\left[E_{l}\right] \lambda_{\varepsilon} \vartheta$ ı y $34 E_{l} \lambda_{\imath} \vartheta \imath \eta$, presentan EI en la segunda sílaba. Lo mismo sucede en otras localidades beocias: $I G$ VII 555,2 (Tanagra) y 3214 (Orcómeno) Eı $\lambda \varepsilon \imath \theta u ı \eta$; en una manumisión de Tisbe, 2228 Eı $\lambda \varepsilon \imath \theta \varepsilon ı \eta$; en Antedón, 4174,3

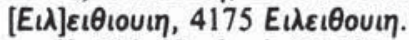

${ }_{16}$ Vid. Heubeck (1972); para las variantes en ático, Threatte (1980: pp. 342-44).
} 
con I y formas también autóctonas con EI. En cualquiera de los dos supuestos, queda excluida la posibilidad de que EI sea una grafia inversa con valor de $/ \mathbf{i} /$.

4. De este análisis de los datos se desprende que, en las manumisiones redactadas en dialecto, no existen grafias inversas EI para escri$\operatorname{bir} / \mathbf{i} /{ }^{17}$. El uso de EI en lugar de I se ha de atribuir a la interferencia de la koiné (para el fenómeno inverso con I dialectal en lugar de EI de la koiné, supra, §2.3). Pese a las abundantes formas en que ello seria posible, EI no nota nunca una /i/ del beocio que corresponda a una /i/ de la variedad supradialectal (en la que, por lo tanto, EI no se justificaría). La única ocasión en que esto sucede es precisamente en una acta

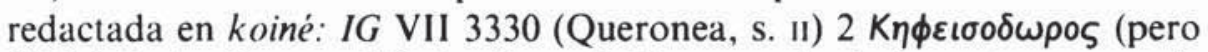

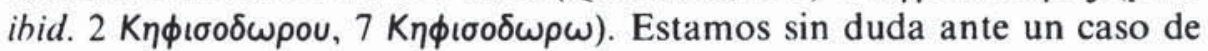
hipercorrección: EI se emplea abusivamente en una palabra con /i/ en el dialecto y en la koiné partiendo de equivalencias tales como beoc. $\varepsilon \pi t:$ koiné $\varepsilon \pi \varepsilon t$, donde la /î/ del beocio y la /ẹ/ (EI) de la koiné remontan a un diptongo /ei/. Cf. otro ejemplo de hipercorrección en otra acta

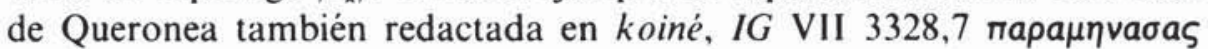

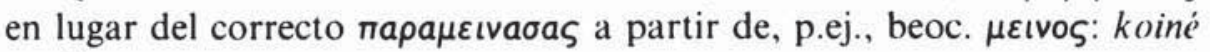

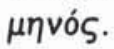

Si queda descartada la posibilidad de que El sea una notación de $/ \mathbf{i} /$, resultante del proceso $/ \overline{\mathrm{u}} />/ \mathbf{i} /$ que postulan los partidarios de la communis opinio, la interpretación más obvia es que el dígrafo tenga su valor habitual en el alfabeto beocio reformado y que represente $/ \bar{e} /$ tal como sugería Schwyzer. Admitido este punto, puesto que un cambio $/ \overline{\mathrm{u}} /$ $>/ \bar{e} /$ es poco plausible desde el punto de vista fonético, resulta inevitable concluir que, al menos en el beocio hablado en la zona en cuestión, el estadio inmediatamente anterior a /ẹ/ era una vocal /ộ/ escrita Y. Es posible que en otras áreas $/ \overline{0} /$ se cerrase en $/ \overline{\mathrm{u}} /$, pero esto es una mera hipótesis no demostrable por el momento.

5. La evolución del diptongo /oi / puede, pues, reconstruirse del siguiente modo: /oi / > [öe] (s. v) > /ộ/ (finales del s. Iv); a fines del s. III, se produce el proceso de deslabialización $/ \overline{\ddot{o}} />/ \bar{e} /$ en Queronea, Lebadea y Coronea ${ }^{1 \mathrm{x}}$. El pretendido paralelismo con el desarrollo de /oi//

\footnotetext{
17 En dos actas de Coronea (Pappadakis 1916) encontramos n. ${ }^{\circ}$, 5-6 провı $\sigma$ -

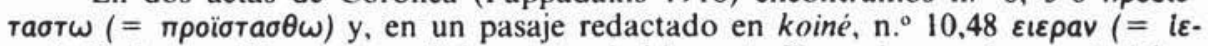
pav). Dado que El nota una /i/ breve (probablemente [j] en el segundo caso), deben ser considerados errores mecánicos no pertinentes para nuestra argumentación.

${ }^{18}$ Carecemos de datos suficientes para saber si la deslabialización de /ộ/ originó
} 
( $/$ oi $/>/ \overline{\ddot{a}} />/ \overline{\mathrm{u}} />/ \ddot{\mathrm{u}} />/ \mathrm{i} /$ ) en griego de época imperial y bizantina es sólo válido para las etapas iniciales ${ }^{19}$. Un paralelo más ajustado a la evolución beocia sería la del diptongo oe del latín clásico confundido con /ẹ/ en latín tardio a través de una etapa intermedia /ộ/ (Allen 1978: p. 62$)^{20}$.

Julián Méndez Dosuna

\section{REFERENCIAS BIBLIOGRÁFICAS}

Allen, William Sidney. 1972: Vox Graeca. A guide to the pronunciation of Classical Greek, (1.0 ed. 1968). Cambridge, Cambridge University Press.

Allen, William Sidney. 1978: Vox Latina. The pronunciation of Classical Latin. (1. ed. 1965). Cambridge, Cambridge University Press.

Bartoněk, Antonin. 1966: Development of the long-vowel system in Ancient Greek dialects. Praga, Státni Pedagogické Nakladatelstvi.

Bechtel, Friedrich. 1921: Die griechischen Dialekte. I. Der lesbische, thessulische, böotische, arkadische und kyprische Dialekt. Berlin, Weidmann.

Beermann, Ernestus. 1876: «De dialecto Boeotica». Georg Curtius, Karl Brugmann (eds.). Studien zur griechischen und lateinischen Grammatik, 9: pp. 1-86. Leipzig, Hirzel (repr. Hildesheim-Nueva York, Georg Olms, 1972).

Blaß, Friedrich. 1881: «Zu griechischen Inschriften». RhM 36: pp. 604-617.

Blümel, Wolfgang. 1982: Die aiolischen Dialekte. Phonologie and Morphologie der inschriftlichen Texte aus generativer Sicht. Göttingen, Vandenhoeck \& Ruprecht.

Brixhe, Claude. 1985: "Énergie articulatoire et phonétique béotienne: faits de substrats ou développements indépendants?" Colloques internationaux du CNRS: "La Béotie antique», pp. 365-84. Paris, Éditions du CNRS.

Brixhe, Claude; Laurent Dubois; René Hodot, Olivier Masson y Guy Vottéro. 1985: "Bulletin de bibliographie thématique et critique: dialectologie-archéologie-histoire", I. "Dialectologie grecque». REG 98: pp. 260-314.

Browning, Robert. 1983: Medieval and Modern Greek.. Cambridge, Cambrige University Press.

Buck, Carl Darling. 1955: The Greek dialects. Grammar, selected inscriptions, glossary. Chicago-Londies, The University of Chicago Press.

Buttenwieser, M. 1911: "Zur Geschichte der böotischen Dialekt». IF 28: pp. 1-106.

Heubeck, Alfred. 1972: "Etymologische Vermutungen zu Eleusis und Eileithya». Kadmos 11: pp. 87-95.

una nueva $/ \bar{e} /$ distinta de la $/ \bar{e} /$ preexistente (que, según hemos visto, estaba evolucionando a /i/) o si, por el contrario, ambas vocales se confundian.

${ }^{19}$ Nótese que el cambio $/ \ddot{\hat{o}} />/ \overline{\mathrm{u}} /$ ha podido ser inducido por la $/ \overline{\mathrm{u}} /(<\mathrm{gr}$. común $/ \bar{u} /$ ) que, a diferencia de lo que sucedia en beocio, existia independientemente en el sistema vocálico de la koiné.

${ }^{20}$ Tengo que agradecer a José Luis Garcia Ramón (Universidad Autónoma de Madrid) y a Martin Peters (Universidad de Viena) el haberme proporcionado copia de algunas publicaciones no fácilmente accesibles. 
Hirt, Hermann. 1912: Handbuch der griechischen Laut- und Formenlehre. . Heidelberg, Winter.

Lejeune, Michel. 1972: Phonétique historique du mycénien et du grec ancien. París, Klincksieck.

Méndez Dosuna, Julián. 1985: Los dialectos dorios del Noroeste. Gramática y estudio dialectal. Salamanca. Ediciones Universidad de Salamanca.

Meister, Richard. 1882: Die griechischen Dialekte auf Grundlage von Ahrens" "De Graecae linguae dialectis», I. Göttingen, Vandenhoeck \& Ruprecht.

Meyer, Gustav. 1896: Griechische Grammatik, Leipzig. Breitkopf \& Härtel.

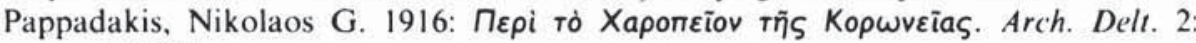
pp. $217-72$.

Rix. Helmut. 1976: Historische Grammatik des Griechischen. Laut-und Formenlehre. Darmstadt, Wissenschaftliche Buchgesellschaft.

Roesch, Paul. 1965: «Notes d'épigraphie béotienne, I. Convention financière entre Thisbé et Chorsiain. $R P h$ 39: pp. 252-56.

Roesch, Paul y John M. Fossey. 1978a: «Neuf actes d'affranchissement de Chéronée». ZPE 1978, 29: pp. 123-37.

Roesch. Paul y John M. Fossey. 1978b: «Un acte d'affranchissement de Coronée en Béotie». ZPE 29: pp. 138-41.

Ruipérez, Martin Sánchez. 1956: «Esquisse d’une histoire du vocalisme grec». Word 12: pp. 67-81.

Sadée, Leopoldus. 1903: De Boeotiae titulorum dialecto. Halle, Niemeyer.

Schmitt, Rüdiger. 1977: Einführung in die griechischen Dialekte. Darmstadt. Wissenschaftliche Buchgesellschaft.

Schwyzer, Eduard. 1939: Griechische Grammatik, I. Allgemeiner Teil. Lautlehre. Worthildung, Flexion. Munich. Beck.

Sturtevant, Edgar H. 1940: The pronunciation of Greek and Latin.. Filadelfia, Linguistic Society of America (repr. Groningen, Bouma's Boekhuis, 1968).

Teodorsson. Sven-Tage. 1974: The phonemic system of the Attic dialect, 400-340 B.C. Gotemburgo-L.und. Acta Universitatis Gothoburgensis.

Threatte, Leslie. 1980: The Grammar of Attic Inscriptions, I. Phonolog.' Berlin-Nueva York, De Gruyter.

Thumb, Albert. 1909: Handhuch der griechischen Dialekte. Heidelberg. Winter.

Thumb, Albert y Anton Scherer. 1959: Handhuch der griechischen Dialekte.. II. Heidelberg, Winter.

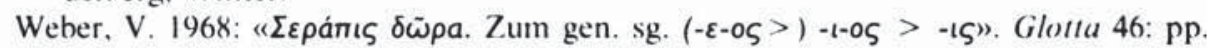
256-63. 